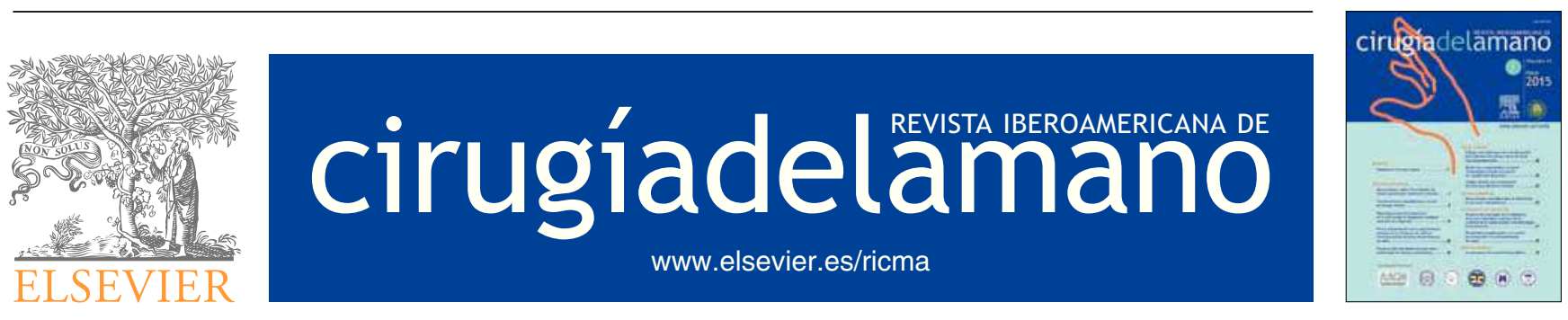

CASO CLÍNICO

\title{
Colgajo microquirúrgico de músculo gracilis para cobertura de palma y dorso de mano tras revascularización
}

\author{
F.J. García-Bernala, ${ }^{a, b}$, P. Zayas ${ }^{a, b}$, J. Regalado y C. Julb ${ }^{\mathrm{b}}$ \\ a Instituto Regalado y Bernal de Cirugía Plástica y de la Mano, Bilbao, España \\ ${ }^{b}$ Hospital Universitario de Basurto, Bilbao, España
}

Recibido el 14 de octubre de 2014. Aceptado el 15 de marzo de 2015.

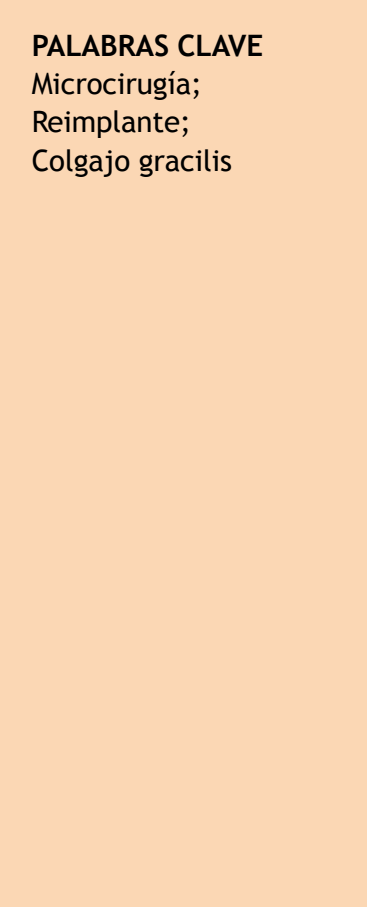

\begin{abstract}
Resumen
Presentamos el caso de un varón de 28 años de edad que sufrió el atrapamiento y la avulsión cutánea de la palma y dorso de la mano izquierda con arrancamiento de todos los pedículos vasculo-nerviosos a la altura del canal carpiano. Presentaba una fractura metafisaria de la falange proximal del quinto dedo, fractura diafisaria del tercer metacarpiano y avulsión de la piel del pulgar a la altura de la articulación trapeciometacarpiana. Las fracturas se fijaron con agujas. Tras la revascularización del pulgar y reparación microquirúrgica de los pedículos avulsionados, el $5 .^{\circ}$ dedo y la piel de la palma y del dorso, evolucionaron a la necrosis. Una semana después se realizó el desbridamiento de la necrosis y la cobertura con un colgajo microvascular de músculo gracilis, anastomosado a la arteria cubital termino-lateralmente. Cinco días después el colgajo se cubrió con un injerto de piel de espesor parcial.

El postoperatorio trascurrió sin complicaciones. El paciente comenzó la rehabilitación a la $4 .^{\text {a }}$ semana tras revascularización. 28 meses tras la intervención presenta un rango de movimiento de los dedos trifalángicos y muñeca completos, rigidez en pulgar (IF $30^{\circ}-90^{\circ}$, MCF $20-60^{\circ}$ ). La sensibilidad era de $20 \mathrm{~mm}$ en el test de discriminación a los dos puntos en los dedos trifalángi$\cos$ y, anestesia en el pulgar. La fuerza de prensa era del 79,6\% $(43 \mathrm{~kg} / 54 \mathrm{~kg})$ respecto a la mano sana.

El colgajo microquirúrgico del músculo gracilis constituye una excelente opción para cobertura en mano, al permitir el deslizamiento de los tendones. Además, mediante el uso de presoterapia, se consigue un excelente resultado estético, en un único tiempo, muy superior al conseguido con colgajos fasciocutáneos.
\end{abstract}

๑ 2015, SECMA. Publicado por Elsevier España, S.L.U. Este es un artículo Open Acces distribuido bajo los términos de la licencia CC BY-NC-ND (http://creativecommons.org/licenses/by-nc-nd/4.0/).

*Autor para correspondencia.

Correo electrónico: fgarciabern@wanadoo.es (F.J. García Bernal).

( $)$ 2015, SECMA. Publicado por Elsevier España, S.L.U. Este es un artículo Open Acces distribuido bajo los términos de la licencia CC BY-NC-ND (http://creativecommons.org/licenses/by-nc-nd/4.0/). 


\section{KEYWORDS}

Microsurgery;

Replantation;

Gracilis muscle
Microsurgical gracilis muscle flap to cover the palm and back of the hand after revascularization

\begin{abstract}
We report the case of a 28 year-old man who suffered a degloving injury of the skin of the palm and dorsum on his left hand, with avulsion of all vessels and nerves in the carpal tunnel. He had fractures of the proximal phalanx of the small finger, on the third metacarpal, and an avulsion of the skin of the thumb at the level of the carpometacarpal joint. The fractures were fixed with Kirschner wires. After microsurgical revascularization and replantation of the thumb, the small finger and the skin of the palm and the dorsum became necrotic. One week later, the necrotic tissue was debrided, and the defect was reconstructed by using a gracilis free flap, end-to-side to the ulnar artery. Five days later the flap was covered with a split skin graft.

The postoperative was uneventful. The patient began rehabilitation at the 4 th week after revascularization. At 28 months after the surgery he had a full range of motion of the triphalangeal fingers and wrist, with some stiffness on his thumb (interphalangeal $30^{\circ}-90^{\circ}$, metacarpophalangeal $20-60^{\circ}$ ). The sensitivity was $20 \mathrm{~mm}$ (two-point discrimination) for triphalangeal fingers and anesthesia in the thumb. The press force was $79.6 \%(43 \mathrm{~kg} / 54 \mathrm{~kg})$ compared to the healthy hand.

The gracilis free flap is an excellent choice for skin coverage of the hand, which allows perfect sliding of the tendons. In addition, by using pressotherapy an excellent outcome can be achieved from a cosmetic point of view in a single stage, far superior to that with fasciocutaneous flaps.

(c) 2015, SECMA. Published by Elsevier España, S.L.U. This is an open access article under the CC BY-NC-ND license (http://creativecommons.org/licenses/by-nc-nd/4.0/).
\end{abstract}

\section{Introducción}

Las lesiones por aplastamiento suponen un reto para el cirujano de la mano. Debido a la naturaleza agresiva de este tipo de traumatismos y a la consiguiente destrucción de múltiples estructuras este tipo de lesiones suelen resultar en un gran compromiso funcional de la extremidad ${ }^{1}$. Por lo general se ven afectadas no solo la piel, que es lo que se aprecia a la simple inspección, sino también la musculatura intrínseca, los tendones, los vasos, los nervios y las estructuras óseas y ligamentosas. De ahí que su tratamiento se convierta en un reto reconstructivo donde el objetivo debe ser, no solo restaurar la anatomía, sino también y fundamentalmente, la función.

\section{Caso clínico}

Se presenta el caso clínico de un varón de 28 años de edad, que sufrió un atrapamiento de mano izquierda con una prensa. A la exploración presentaba avulsión cutánea del dorso y palma de la mano izquierda, sección de todos los pedículos vasculonerviosos a la altura del pliegue de la muñeca y avulsión de la piel del pulgar a la altura de la muñeca (figs. $1 \mathrm{~A}$ y $1 \mathrm{~B}$ ). En la radiografía presentaba una fractura metafisaria de la falange proximal del $5 .^{\circ}$ dedo y una fractura metafisaria del 3. ${ }^{\text {er }}$ metacarpiano.

Bajo anestesia regional se realizó el desbridamiento de las estructuras desvitalizadas y fijación de las fracturas con agujas de Kirshcner. A continuación se procedió a la reparación microquirúrgica de los pedículos vasculonerviosos (ra-
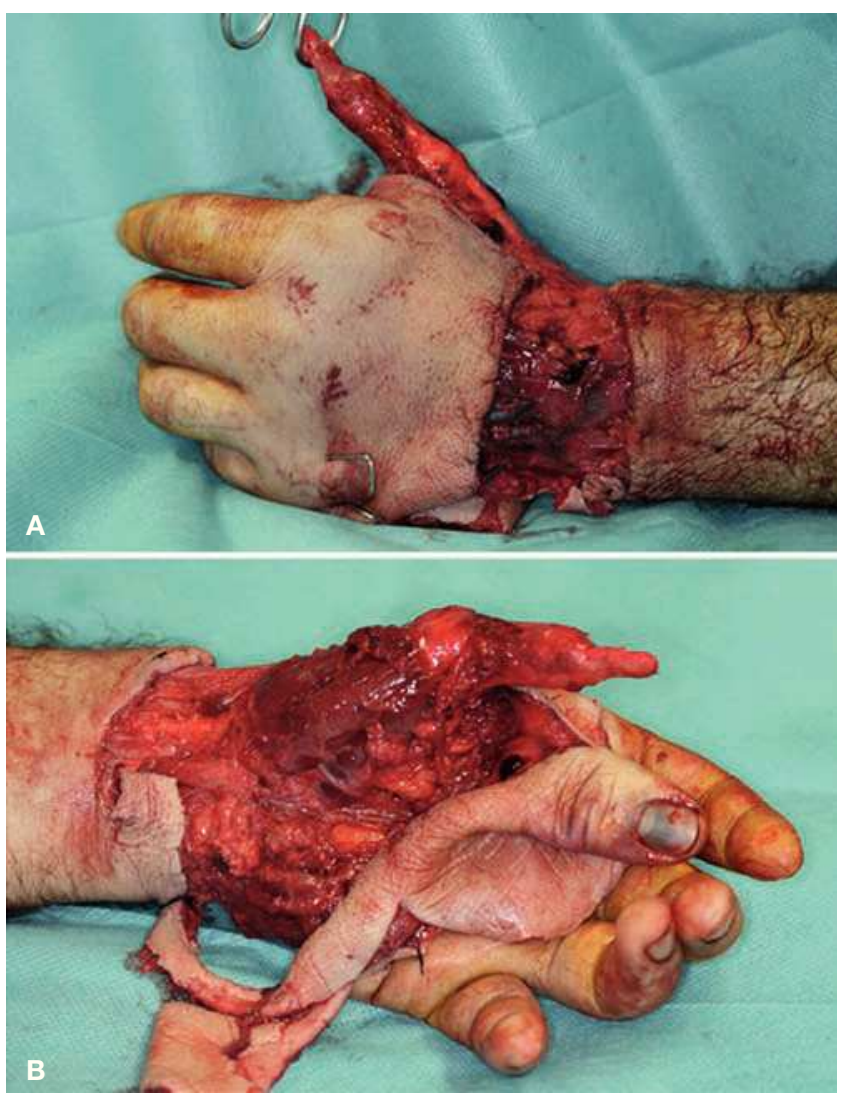

Figuras 1A y 1B Avulsión cutánea de dorso y palma de mano izquierda y del pulgar a la altura de la articulación metacarpofalángica. 
dial del índice y segundo, tercero y cuarto comisurales), al reimplante de la avulsión cutánea del pulgar mediante anastomosis termino-terminal de la arteria colateral cubital y a la neurorrafia de ambos nervios colaterales. La piel del dorso y de la palma, se repuso con una sutura sin tensión y se dejó un drenaje no aspirativo.

Catorce horas después, el quinto dedo presentó signos de isquemia arterial, por lo que se revisó. Se comprobó la existencia de una trombosis de la arteria del $4 .^{\circ}$ espacio comisural, y tras la limpieza y revisión se revascularizó el dedo. Sin embargo, veinticuatro horas después, tanto el quinto dedo como la piel del dorso y palma de la mano comenzaron a mostrar signos de isquemia. Cinco días después, el paciente presentaba una clara necrosis de la piel del dorso y de la palma, así como del quinto dedo (figs. 2A y 2B). Una vez delimitada la necrosis, a la semana del accidente, se realizó un nuevo desbridamiento de la piel de la palma y del dorso de la mano, se realizó la amputación del $5 .^{\circ}$ radio (figs. $3 \mathrm{~A}$ y $3 B$ ) y la cobertura con un colgajo microvascular del músculo gracilis revascularizado mediante anastomosis termino-lateral a la arteria cubital (fig. 4). El paciente quedó ingresado con tratamiento analgésico, antibiótico y profilaxis antitrombótica. Cinco días después, el colgajo se cubrió con un injerto de piel parcial, siendo dado de alta al día siguiente.

El paciente comenzó la rehabilitación a la cuarta semana tras la revascularización, tras haber epitelizado correctamente todas las heridas, precisando del empleo de férulas con elásticos para vencer la rigidez de metacarpofalángicas y de presoterapia para reducir el edema del colgajo.
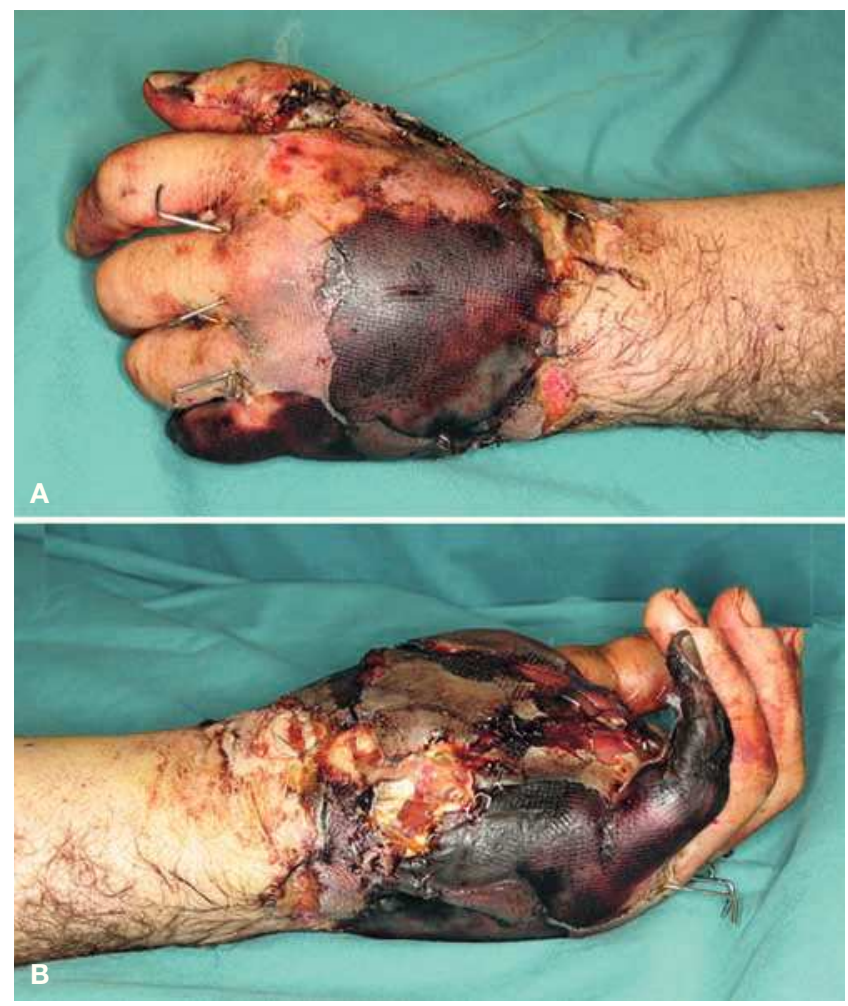

Figuras $2 \mathrm{~A}$ y $2 \mathrm{~B}$ Necrosis del $5 .^{\circ}$ dedo y de la piel de la palma y el dorso de la mano.
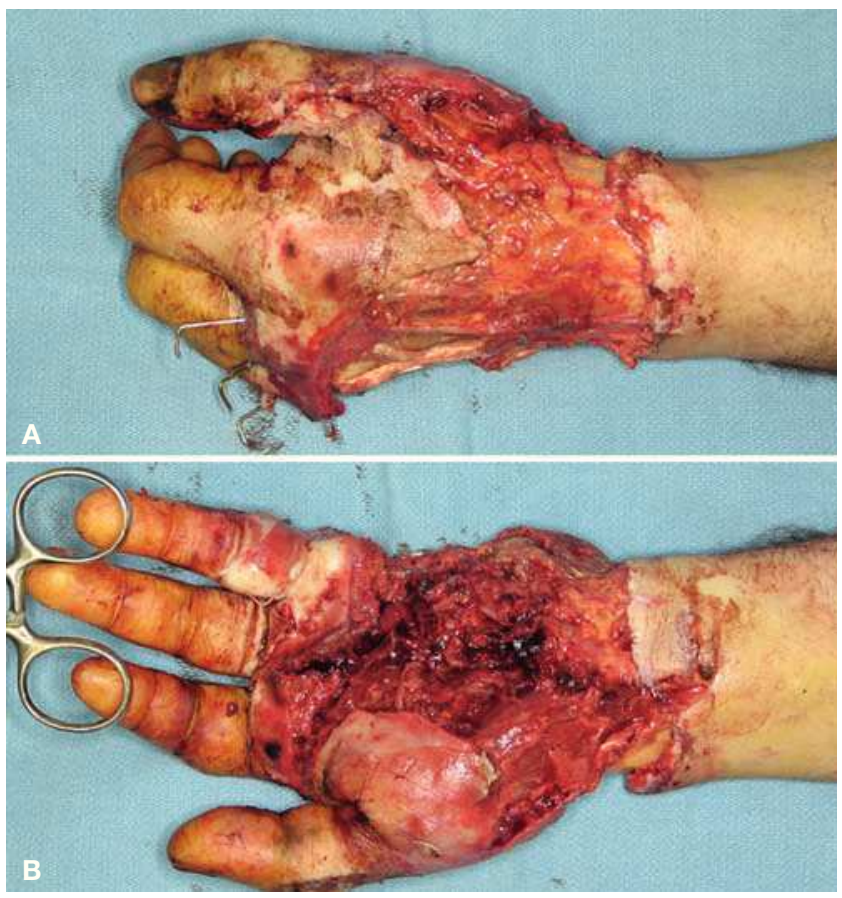

Figuras $3 \mathrm{~A}$ y $3 \mathrm{~B}$ Aspecto de la mano tras el desbridamiento de la piel de la palma y del dorso de la mano y la amputación del $5 .^{\circ}$ radio.

El postoperatorio transcurrió sin complicaciones observando en todo momento una correcta perfusión del colgajo, del reimplante del pulgar y del resto de dedos.

Veintiocho meses después del traumatismo el paciente presenta un rango de movilidad de los dedos trifalángicos y muñeca completos, rigidez en el pulgar (IF $30^{\circ}-90^{\circ}$ y MCF $\left.20^{\circ}-60^{\circ}\right)$, sensibilidad de 20 pd en dedos trifalángicos y anestesia en el pulgar con fuerza de prensa del 79\% (43 kg/54 kg) con respecto a la mano sana (figs. $5 \mathrm{~A}$ y $5 \mathrm{~B}$ ).

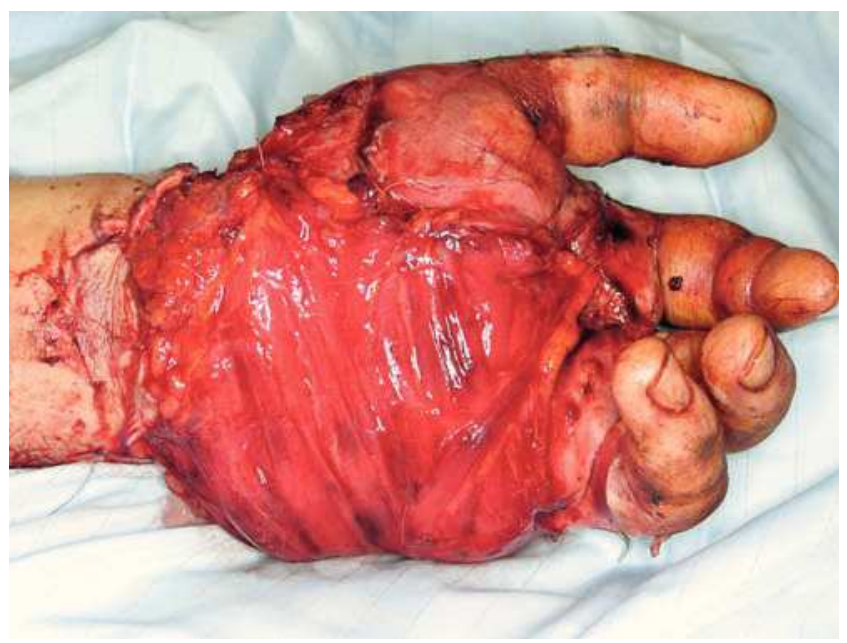

Figura 4 Cobertura de las estructuras nobles expuestas con un colgajo microvascular de músculo gracilis revascularizado mediante anastomosis término-terminal a la arteria cubital. 

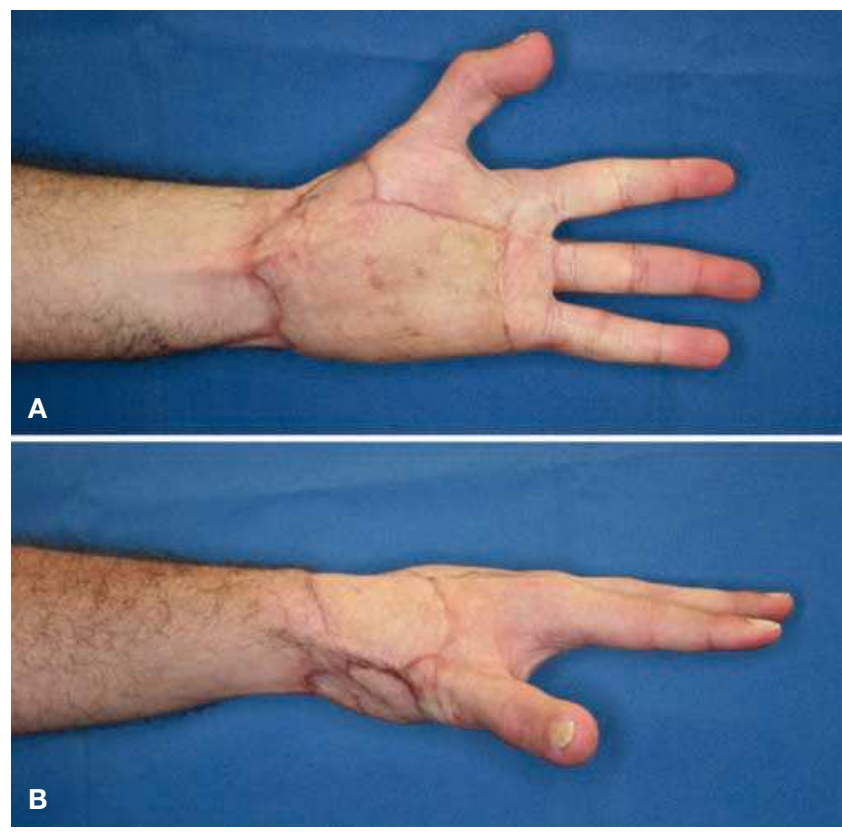

Figuras 5A y 5B Resultado estético a los 28 meses del traumatismo.

\section{Discusión}

Los principios fundamentales para el tratamiento de una herida en la mano son idénticos a los del tratamiento del resto de las heridas. Tras haber asegurado la estabilidad hemodinámica del paciente, es prioritario realizar un desbridamiento agresivo de todo el material necrótico y no viable $^{2,3}$, seguido de una adecuada cobertura antibiótica y antitetánica.

En ocasiones, la naturaleza del traumatismo nos hace enfrentarnos a tejidos de viabilidad indeterminada, altamente contaminados o en los que un desbridamiento agresivo inicial conlleva una pérdida de estructuras y de función inasumibles. En estas situaciones, los desbridamientos seriados juegan un destacado papel. Mediante desbridamientos sucesivos en los días siguientes al traumatismo, se puede evaluar y definir la viabilidad o no de esos tejidos. En estos casos, la cobertura puede diferirse para asegurar la ausencia de signos de infección o la presencia de estructuras desvitalizadas que posteriormente puedan comprometer la reconstrucción. Y esta situación no es infrecuente en el caso de los traumatismos de alta energía, como los aplastamientos.

No obstante, lo ideal sería hacer todo en un primer tiempo, siempre y cuando las condiciones lo permitan ${ }^{2,3}$. Así, tras el desbridamiento, se realizará la fijación de las fracturas, la reparación de las estructuras ligamentosas, tendinosas y vasculonerviosas, y se procederá al cierre de las heridas. Si existiera un defecto de partes blandas con exposición de estructuras nobles, se aportará una cobertura precoz con tejido vascularizado. De este modo se reducirá la morbilidad postoperatoria, el riesgo de infección y el número de cirugías posteriores. Como consecuencia, se facilitará el ini-
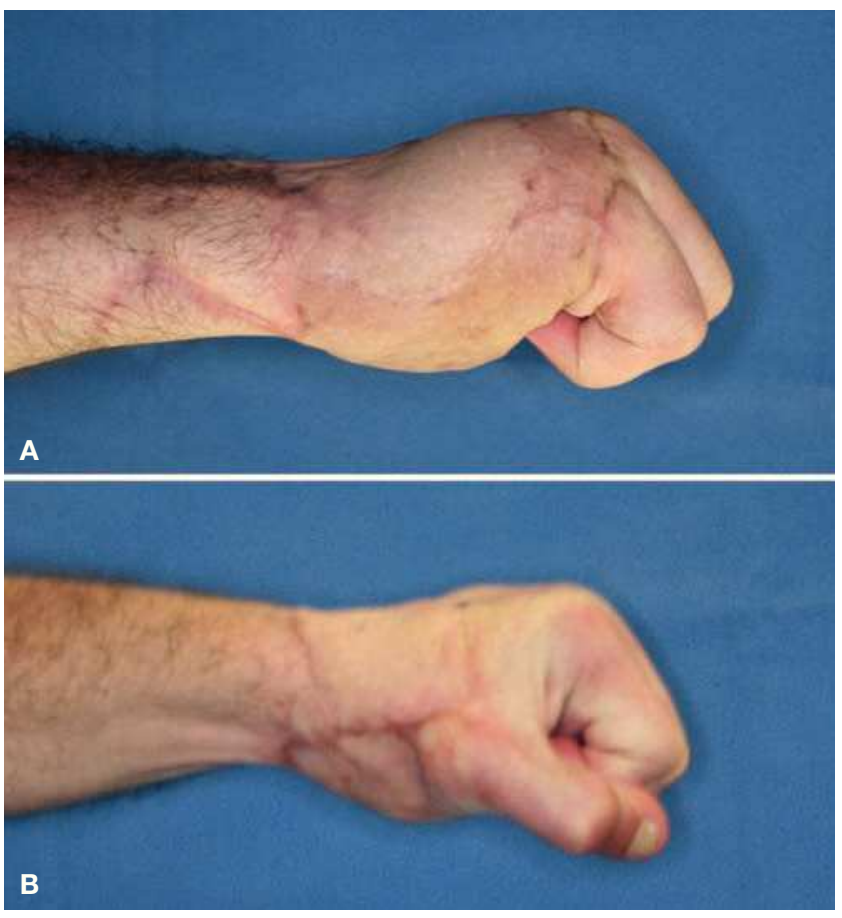

Figuras 6A y 6B Resultado funcional a los 28 meses del traumatismo.

cio de una rehabilitación precoz mejorando así el resultado funcional final ${ }^{3-7}$.

Como opción terapéutica para la cobertura del caso que nos interesa, existen distintas opciones terapéuticas. En este caso, dada la extensión del defecto, de entre los colgajos de menor morbilidad y que se pudiese adaptar a nuestras necesidades, se optó por la transferencia microvascular del músculo gracilis.

El gracilis es un músculo acintado, localizado superficialmente en la cara medial del muslo. El fácil acceso a él, su anatomía constante, la reducida morbilidad ${ }^{8,9}$ y la posibilidad de trabajar a dos campos, lo convierte en uno de los colgajos de elección para este tipo de defectos. Y si bien están citados como inconvenientes la escasa longitud de su pedículo o el escaso volumen-superficie aportado, también están ya descritos ciertos detalles técnicos ${ }^{10}$ que solventan esta situación. No obstante, algunos autores, optan por el empleo de colgajos fasciocutáneos para este tipo de defectos en mano por una hipotética mayor permisibilidad al deslizamiento tendinoso de los tendones frente a los colgajos musculares ${ }^{11}$. Sin embargo, nuestra experiencia con este colgajo, en este y en otros casos similares nos demuestra que los tendones se deslizan sin ningún tipo de compromiso bajo el músculo. Finalmente, otro de los clásicos inconvenientes de los colgajos musculares, es el exceso de volumen aportado y el pobre resultado estético. Sin embargo, este exceso de volumen se puede reducir fácilmente con el uso de presoterapia, logrando coberturas, evitando así, la necesidad de cirugías posteriores para adelgazar el colgajo ${ }^{12,13}$.

El colgajo microquirúrgico del músculo gracilis constituye una excelente opción en los defectos en mano, tanto en dorso como en palma, permitiendo el deslizamiento de los 
tendones. Además, mediante el uso de presoterapia, se consigue un excelente resultado estético, en un único tiempo, muy superior al conseguido con colgajos fasciocutáneos.

\section{Conflicto de intereses}

Los autores declaran no tener ningún conflicto de intereses.

\section{Bibliografía}

1. Del Piñal F, Pisani D, García-Bernal FJ, Regalado J, Del Pino FJ, Ayala $\mathrm{H}$. Massive hand crush: the role of a free muscle flap to obliterate the dead space and to clear deep infection. J Hand Surg [Br]. 2006;31:588-92.

2. Haury B, Rodeheaver G, Vensko J, Edgerton MT, Edlich RF. Debridement: an essential component of traumatic wound care. Am J Surg. 1978;135:238-42.

3. Scheker LR, Ahmed O. Radical debridement, free flap coverage, and immediate reconstruction of the upper extremity. Hand Clin. 2007;23:23-36.

4. Godina M. Early microsurgical reconstruction of complex trauma of the extremities. Plast Reconstr Surg. 1986;78:285-92.
5. Lister G, Scheker L. Emergency free flaps to the upper extremity. J Hand Surg Am. 1988;13:22-8.

6. Ninkovic M, Deetjen H, Ohler K, Anderl H. Emergency free tissue transfer for severe upper extremity injuries. J Hand Surg [Br]. 1995;20:53-8.

7. Giessler GA, Erdmann D, Germann G. Soft tissue coverage in devastating hand injuries. Hand Clin. 2003;19:63-71.

8. Mathes SJ, Nahai F. Classification of the vascular anatomy of muscles: experimental and clinical correlation. Plast Reconstr Surg. 1981;67:177-87.

9. Giordano PA, Abbes M, Pequignot JP. Gracilis blood supply: anatomical and clinical re-evaluation. $\mathrm{Br} J$ Plast Surg. 1990;43:266-72.

10. Piñal Matorral F, García Bernal FJ, Delgado Martínez J, Gómez Jorge C, Arenas Bermejo E. Optimización del colgajo de Gracilis libre. Rev Cir Plast Iberolatinoam. 2004;30:77-86.

11. Scheker LR, Kleinert HE, Hanel D. Scheker LR, Kleinert HE, Hanel DP. Lateral arm composite tissue transfer to ipsilateral hand defects. J Hand Surg [Br]. 1987;12:665-72.

12. Tropet Y, Brientini JM, Garbuio P, Ridoux PE, Vichard P. Reconstruction of a complex defect of the dorsum of the hand. $J$ Hand Surg [Br]. 1995;20:591-5.

13. Pivato G, Cortese P, Novelli C, Pegoli L, Pajardi G. Muscular flaps for coverage of soft tissue defects of the hand. En: Dupert T, Georgescu A, Soucacos PN, editores. Primary care of complex injuries of the hand and wrist. 2010 FESHH Instructional Course Book. Athens: Konstantaras Medical Publications; 2010. 